\title{
DIVERGÊNCIAS ENTRE LEGISLAÇÕES DO DIMENSIONAMENTO DE ENFERMAGEM EM UNIDADES DE TERAPIA INTENSIVA
}

Maria Auxiliadora Rodrigues ${ }^{1}$, Rodolfo César Cardoso de Paula ${ }^{2}$, Rosimere Ferreira Santana ${ }^{2}$

Objetivo: discutir as divergências entre as legislações vigentes sobre o dimensionamento de enfermagem e as suas repercussões na assistência em unidades de terapia intensiva. Metodologia: estudo reflexivo sobre as fiscalizações realizadas pelo COREN-RJ nas unidades de terapia intensiva a partir de 2010, com parâmetro nas Resoluções do COFEN e Anvisa. Resultados: comparados os dispositivos legais e as implicações ao exercício profissional, fica evidenciado que a Anvisa descumpre a lei na atividade privativa do enfermeiro ao paciente crítico. Conclusão: a RDC Anvisa lesa a lei da enfermagem e compromete a qualidade da assistência de enfermagem ao paciente crítico.

Descritores: Enfermagem, Recursos Humanos de Enfermagem no Hospital, Unidades de Terapia Intensiva, Legislação de Enfermagem.

\section{DIFFERENCES BETWEEN LEGISLATION OF NURSING SCALING IN INTENSIVE CARE UNITS}

Objective: To discuss the differences between current legislation in nursing scaling and its repercussions in the assistance in intensive care units. Methodology: Reflective study on the inspections carried out by COREN-RJ in the intensive care units from 2010, with parameters of COFEN and ANVISA Resolutions. Results: Compared with the legal devices and the implications to the Professional Exercise, it was evident that ANVISA disregarded the law in the exclusive activity of the Nurse to the critical patient. Conclusion: RDC ANVISA harms the nursing law and compromises the quality of nursing care to critical patients.

Descriptors: Nursing, Human Resources of Nursing Hospital, Intensive Care Units; Legislation, Nursing.

\section{LAS DIVERGENCIAS ENTRE LAS LEGISLACIONES DIMENSIONAMIENTO DE ENFERMERÍA EN UNIDADES DE CUIDADOS INTENSIVOS}

Objetivo: Discutir las diferencias entre la legislación vigente en materia de diseño de enfermería y su impacto en la atención en unidades de cuidados intensivos. Metodología: Estudio reflectante en las inspecciones realizadas por COREN-RJ en unidades de cuidados intensivos a partir de 2010, con las resoluciones de parámetros COFEN y ANVISA. Resultados: disposiciones e implicaciones de la práctica profesional jurídicos, en comparación, evidenciaron que la ANVISA viola la ley sobre la actividad privada para cuidar a pacientes en estado crítico. Conclusión: RDC ANVISA daña la ley de enfermería y compromete la calidad de la atención de enfermería a pacientes en estado crítico.

Descriptores: Enfermería, Personal de Enfermería em Hospital, Unidade de Cuidados Intensivos, Legislación de Enfermería.

IEnfermeira. Conselho Regional de Enfermagem do Rio de Janeiro ( Coren-RJ).

2Enfermeiro(a). Universidade Federal Fluminense (UFF). E-mail: rodolphodepaula@hotmail.com 


\section{INTRODUÇÃO}

O dimensionamento de pessoal de enfermagem é definido como um processo sistemático, que fundamenta o planejamento e a avaliação do quantitativo e qualitativo de profissionais necessários para prover a assistência, de acordo com a singularidade dos serviços de saúde, que garantam a segurança dos usuários e dos trabalhadores ${ }^{(1)}$.

O dimensionamento de recursos humanos é uma atividade/habilidade gerencial do enfermeiro, que envolve a previsão de pessoal sob os enfoques quantitativo e qualitativo, com vistas ao atendimento das necessidades de assistência ao paciente, na busca de uma melhor qualidade possivel da atenção(2). $O$ artigo $2^{\circ}$ da Resolução COFEN $n^{\circ}$ 293/2004 dispõe que o dimensionamento e a adequação quantiqualitativa do quadro de profissionais de enfermagem devem basear-se em características relativas à instituição, ao serviço de enfermagem e à clientela(3).

A Unidade de Terapia Intensiva (UTI) é uma área crítica destinada à internação de pacientes graves, que requerem atenção profissional especializada de forma continua, materiais específicos e tecnologias necessárias ao diagnóstico, monitorização e terapia $^{(4)}$.

Nesse sentido, subentende-se que se trata de um local especializado e tecnológico, identificado como espaço laboral destinado a profissionais da saúde, principalmente médicos e enfermeiros, que necessitam de preparo especializado, pois, invariavelmente, podem se defrontar com situações cujas decisões definem o limite entre a vida ou a morte das pessoas.

A metodologia de dimensionamento é um instrumento valioso para o alcance de parâmetros mínimos para suprir as necessidades dos pacientes, com vistas à melhoria da qualidade da assistência e satisfação da equipe nas atividades diárias $^{(5)}$

O Conselho Federal de Enfermagem (COFEN), por

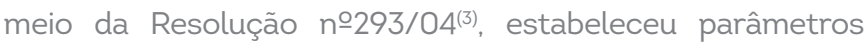
para dimensionar o quantitativo mínimo para cobertura assistencial nas instituições de saúde, com base nas características relativas à organização, ao serviço de enfermagem e à clientela.

Essa fora revogada pela Resolução COFEN 527/2016(6), que considera a mesma forma de cálculo de profissionais nas unidades de internação, porém, em seu artigo 3으, considera para efeito de cálculo o valor de 18 horas de enfermagem por paciente na assistência intensiva, ou seja, de pacientes graves. A Resolução estabelece, ainda, a distribuição percentual do total de profissionais em pacientes graves ou assistência de intensiva são enfermeiros 52\% do total calculado(6)

Porém, observa-se que atualmente as unidades de terapia intensiva adotam o quantitativo recomendado por outras legislações não sendo o estipulado pelo Sistema COFEN/ COREN. Tais legislações não levam em consideração o grau de complexidade dos pacientes assistidos nessas unidades, estipulando meramente um número absoluto de leitos ao número reduzido de profissionais, sendo desconsideradas as atividades de cada elemento pertencente à categoria de enfermagem na assistência ao paciente crítico.

A problemática vivenciada como enfermeiro fiscal do Conselho Regional de Enfermagem do Rio de Janeiro motivounos a realizar este estudo sobre essas divergências legais em relação ao dimensionamento de pessoal de enfermagem nas unidades de terapia intensiva. Dessa forma, delimita-se como problema de estudo: de quem é a competência legal em determinaro dimensionamento de enfermagem nas unidades de terapia intensiva?

Diante dessas considerações, a presente reflexão tem como objetivo: discutir as divergências entre as legislações vigentes sobre o dimensionamento de enfermagem e as suas repercussões na assistência em unidades de terapia intensiva.

\section{METODOLOGIA}

Estudo reflexivo sobre as fiscalizações realizadas pelo Conselho Regional de Enfermagem do Rio de Janeiro nas unidades de terapia intensiva a partir do ano de 2010.

A fiscalização se embasa na Resolução COFEN 374/2011 que regulamenta o processo de fiscalização em todo território nacional( ${ }^{(7)}$, sendo utilizado como parâmetro de cálculo para o dimensionamento de pessoal de enfermagem a Resolução COFEN 293/2004 em todas as áreas de atuação da enfermagem.

Após a análise das fiscalizações geradas e os cálculos de dimensionamento de pessoal com base na Resolução COFEN e o dimensionamento proposto pela RDC 26 ANVISA utilizado pelas instituições, foi realizada uma análise comparativa desses instrumentos legais da ANVISA e do COFEN e 
buscou-se compreender qual o resultado desse diferencial na assistência.

\section{REFLEXÃO SOBRE OS DISPOSITIVOS LEGAIS QUE DISPÕEM SOBRE O DIMENSIONAMENTO DE PESSOAL DE ENFERMAGEM}

A Lei 7498 de 25 de junho de 1986 regulamenta o exercício profissional de enfermagem e é o instrumento legal que legisla sobre a atuação dos profissionais de enfermagem e descreve em seu texto no, artigo 110, como atividade privativa do enfermeiro os cuidados diretos de enfermagem a paciente graves com risco de vida(8).

Segundo o descrito, a assistência direta ao paciente crítico é privativa ao enfermeiro e o técnico de enfermagem tem como função assistir ao enfermeiro na prestação de cuidados diretos a pacientes em estado grave, conforme o artigo 10 inciso I alínea b do Decreto Lei 94406 de 08 de junho de $1987^{(9)}$.

\begin{tabular}{cll} 
Nesse & \multicolumn{2}{c}{ sentido, as } \\
resoluções & da & Vigilância \\
Sanitária & e & portarias
\end{tabular}
ministeriais ferem diretamente a Lei $7498 / 86$, pois não contemplam tal atividade privativa conferida ao enfermeiro, sendo constatado em seu texto um quantitativo de enfermeiros inferior ao necessário para uma prestação com segurança, qualidade e legalidade.

Contextualizando, a Lei $7498 / 86$ não possui artigos específicos demonstrando o número exato de profissionais de enfermagem/leito, até porque essa avaliação deve ser feita pela complexidade e grau de cuidados que esse deve receber. Já o Conselho Regional de Enfermagem possui uma Resolução que proporciona - cálculo de profissionais de enfermagem levando em consideração o tipo de cliente a ser assistido e o número de horas de enfermagem necessário para essa assistência.

Em contrapartida, a RDC Anvisa 26, de 11 de maio de 2012, que altera a RDC Anvisa 7/2010 - que estabelecia o quantitativo de 01 enfermeiro para cada 08 leitos e fração por turno, 01 técnico de enfermagem para cada 02 leitos e fração e $\mathrm{Ol}$ técnico de enfermagem de apoio por turno passa a considerar um quantitativo mínimo de $\mathrm{Ol}$ enfermeiro para cada 10 leitos e fração e 01 técnico de enfermagem para cada 02 leitos e fração, por turno ${ }^{(10)}$. Nesse sentido, não se considera o recomendado pela Resolução COFEN 527, que avalia primeiramente para se determinar o quantitativo de profissionais o grau de dependência e de cuidado de enfermagem necessário para o cliente em questão.

A Resolução COFEN 527/2004, em seu artigo 3ㅇ, considera para efeito de cálculo o valor de 18 horas de enfermagem por paciente na assistência intensiva, ou seja, de pacientes graves. Estabelece a distribuição percentual do total de profissionais em pacientes graves ou assistência intensiva, sendo que os enfermeiros devem corresponder a $52 \%$ do total calculado(6). Por ser atividade privativa do enfermeiro a assistência direta a pacientes graves, o número de enfermeiros deve ser superior ao de técnicos de enfermagem nesses setores onde ocorre assistência a pacientes graves.

Cabe ressaltar que as unidades de terapia intensiva são destinadas aos pacientes graves que necessitam de cuidados especializados de enfermagem e médico. É fato que atividades de enfermagem executadas nesses locais requerem conhecimento científico e tomada de decisão, sendo privativo ao enfermeiro, conforme Lei 7498/86 artigo 110 - Cuidados diretos de Enfermagem a pacientes graves com risco de vida, e cuidados de Enfermagem de maior complexidade técnica e que exijam conhecimentos de base científica e capacidade de tomar decisões imediatas ${ }^{(8)}$.

Essas discordâncias legais causam diferenças no quantitativo de profissionais que podem refletir diretamente no cuidado prestado.

Por exemplo, em uma unidade de terapia intensiva de 10 leitos em que a jornada semanal é de enfermagem de 40 horas, o cálculo de profissionais seria desta forma:

$\mathrm{OP}=\mathrm{Km} \times \mathrm{THE}$

$\mathrm{THE}=\mathrm{nO}$ pacientes (leitos) $\times \mathrm{nO}$ horas de enfermagem

THE $=10 \times 18=180$ horas de enfermagem

$\mathrm{Km}=\underline{\mathrm{DS}} \times \mathrm{IST}=\underline{7 \times 1,15}=0,2012$

JST $\quad 40$

$\mathrm{QP}=0,2012 \times 180=36,21$ profissionais de enfermagem

$52 \%$ são enfermeiros, então 19 são enfermeiros e 17 são técnicos de enfermagem.

Se dividirmos na escala de revezamento de enfermagem 12x36h, ou seja, 02 plantões dias e dois plantões noite, teríamos: 
Quadro 1 - Escala de Revezamento 12x36 horas

\begin{tabular}{lccccc} 
Categoria & SD O1 & SN O1 & SD 02 & SN 02 \\
Enf, Diarista & & \multicolumn{2}{c}{3} & \\
$\begin{array}{l}\text { Téc. Diarista } \\
\text { Enfermeiros }\end{array}$ & 4 & 4 & & 4 & 4 \\
$\begin{array}{l}\text { Técnicos de } \\
\text { enfermagem }\end{array}$ & 4 & 4 & & 4 & 4 \\
\hline
\end{tabular}

Seria uma proporção de $\mathrm{Ol}$ enfermeiro para 2,5 leitos e 01 técnico de enfermagem para 2,5 leitos. Na mesma unidade de 10 leitos para uma jornada de 40 horas teríamos no quadro de enfermagem o quantitativo de acordo RDC 26/2012 05 enfermeiros e 20 técnicos de enfermagem, sendo uma proporção de $\mathrm{Ol}$ enfermeiro para 10 leitos e $\mathrm{Ol}$ técnico de enfermagem para $\mathrm{O} 2$ leitos.

Quadro 02 - Quadro comparativo do quantitativo de profissionais para 10 leitos com jornada de 40 horas

\begin{tabular}{lll} 
Categoria & RDC 26/2012 & Resolução 293 \\
Enfermeiro & 05 & 19 \\
Téc. de enfermagem & 20 & 17 \\
\hline
\end{tabular}

Assim, segundo a RDC, o total seria de 25 profissionais de enfermagem, sendo 05 deles enfermeiros, e segundo a Resolução 527, o total seria de 36, sendo 19 desses enfermeiros.

Cabe ressaltar que a Resolução COFEN 527 é o instrumento legal utilizado pelo COFEN para determinar o dimensionamento de enfermagem de acordo com a o grau de complexidade e de cuidados de enfermagem aos clientes assistidos. Outro ponto é o fato de que esse instrumento de cálculo prevê o índice de segurança técnica (IST) de 15\%, conforme o artigo 10으, para cobertura do absenteísmo e das ausências por benefícios.

O dimensionamento de pessoal, visto sob essa ótica, representa um instrumento/estratégia que pode minimizar o absenteísmo na enfermagem. Além da função de proteção ao cliente/usuário, aumenta a segurança do trabalhador porque, ao prever o índice de segurança, parte do planejamento desse instrumento, realiza o acréscimo necessário para cobrir os imprevistos que podem ocorrer com a equipe de enfermagem ao longo das 24 horas de trabalho(11)

O absenteísmo é um fator causador de problemas para a equipe de enfermagem, visto que a ausência de um profissional na equipe sobrecarrega o trabalho dos demais, exigindo um ritmo mais acelerado e um volume maior de trabalho no processo de cuidar do cliente. Essa sobrecarga poderá prejudicar a saúde do trabalhador, ocasionando desgaste físico, psicológico e social; e, como consequência, o adoecimento. Evidencia-se que o problema do absenteísmo pode desencadear em cascata 0 adoecimento dos trabalhadores de enfermagem ${ }^{(11)}$.

A elevada carga de trabalho exigida pelo paciente internado na UTI, decorrente da instabilidade hemodinâmica e da necessidade de cuidados de enfermagem contínuos, deve ser considerada como informação fundamental para o dimensionamento e para alocação de recursos humanos, sobretudo quando se busca maior segurança para o profissional e qualidade do serviço ofertado ${ }^{(12)}$, aspecto não contemplado pela RDC 26, pois avalia friamente o profissional e a quantidade de leitos.

Outro fator a se comparar é o fato da RDC Anvisa 26/2012 não apresentar no seu escopo a metodologia que originou o valor do quantitativo estipulado de profissionais de enfermagem por leito. Já a Resolução COFEN 293 apresenta no seu anexo a metodologia que determinou o número de horas de enfermagem por grau de complexidade, sendo ajustados pela Resolução COFEN 527, ou seja, acredita-se que a RDC Anvisa 26 impôs um quantitativo sem avaliar o cuidado e a necessidade do cliente assistido nas unidade de terapia intensiva.

Ao se comparar o quantitativo de profissionais de enfermagem no exemplo acima, estipulado pela Resolução COFEN 527 (19 enfermeiros) em relação ao da RDC Anvisa 26/2012 (05 enfermeiros) observa-se um aumento do quadro de enfermeiros na assistência ao paciente crítico como preconizado pela Lei 7498/86.

Existe ainda uma tendência de atribuir os altos custos da saúde aos gastos com o quadro de pessoal. Como exemplo temos a redução de custos, que pode recair sobre a equipe de enfermagem, ocasionando diminuição do quadro de pessoal, o que repercute na qualidade da assistência prestada. ${ }^{(13)}$

Questiona-se se essas diferenças de quantitativo não estarão relacionadas meramente aos possiveis gastos com a contratação de funcionários, ou seja, a qualidade do serviço prestado não é o primeiro ponto a ser avaliado.

A fiscalização do Conselho Regional de Enfermagem do Rio de Janeiro age contra o déficit de profissionais, pois realiza os cálculos de dimensionamento de pessoal com base na Resolução do COFEN em todas as áreas onde ocorre o exercício profissional de enfermagem e enfatiza a necessidade da presença do enfermeiro, principalmente na assistência ao paciente crítico e orienta os enfermeiros responsáveis técnicos no cumprimento das legislações de enfermagem.

As ações fiscais são finalizadas com procedimentos jurídicos pelo COREN-RJ no intuito de corrigir o déficit encontrado nos cálculos realizados decorrentes das 
fiscalizações. A limitação deste estudo é que não se pode determinar o atual déficit de enfermeiros nas unidades de terapia intensiva do estado do Rio de Janeiro.

\section{CONCLUSÃO}

A realização deste estudo possibilitou visualizar os parâmetros preconizados pela Resolução COFEN 293 e as discrepâncias em relação à RDC Anvisa 26/2012, além do prejuízo causado por essa legislação que contraria a Lei do exercício profissional em relação à atividade privativa do enfermeiro no cuidado do paciente crítico.

No que diz respeito à RDC Anvisa 26, a proporção de enfermeiros é inferior ao de técnicos de enfermagem, o que ainda está distante do que é preconizado pelo COFEN, configurando-se, assim, um conflito para a enfermagem brasileira.

Acredita-se que a partir desta reflexão seja possivel que os enfermeiros percebam os prejuízos à categoria de enfermagem, à sociedade e principalmente à qualidade da assistência de enfermagem ao paciente crítico.
REFERÊNCIAS

1. Fugulin FMT, Rossetti AC, Ricardo CM, Possari JF, Mello MC, Gaidzinski RR. Tempo de assistência de enfermagem em Unidade de Terapia Intensiva: avaliação dos parâmetros propostos pela Resolução COFEN n293/04. Rev. Latino-Am. Enfermagem [Internet] 2012 [cited 2014 June 10];20(2):[09 telas] Available from: www.scielo.br/pdf/rlae/v20n2/pt_15.pdf

2. Inoue KC, Matsuda LM. Dimensionamento da equipe de enfermagem em unidade de terapia intensiva para adultos. Rev. Acta Paul Enferm [Internet]. 2010 [cited 2014 June 10]: 23(3):379-84. Available from: http://www.scielo. br/pdf/ape/v23n3/v23n3all

3. Conselho Federal de Enfermagem (COFEN). Resolução COFEN $n^{\circ}$ 293, de 21 de setembro de 2004. Fixa e estabelece parâmetros para o dimensionamento do quadro de profissionais de enfermagem nas unidades assistenciais das instituições de saúde e assemelhados [Internet]. 2004 [cited 2014 dec 12]. Available from: http://cofen.gov.br/resoluocofen-2932004_4329.html

4. Agência Nacional de Vigilância Sanitária. Resolução Da Diretoria Colegiada. RDC no 7 de 24 de fevereiro de 2010. Dispõe sobre os requisitos minimos para funcionamento de unidades de terapia intensiva e da outras providências. [Internet]. 2010 [cited 2014 June 10]. Available from: http:// bvsms.saude.gov.br/bvs/saudelegis/anvisa/2010/res0007_24_02_2010. html

5. Inoue KC, Matsuda LM. Dimensionamento da equipe de enfermagem da UTI-adulto de um hospital de ensino. Rev. Eletr. Enf. [Internet]. 2009 [cited 2014 June 10]:11(1):55-63. Available from: http://www.fen.ufg.br/revista/ vll/nl/vllnla07.htm

6. Conselho Federal de Enfermagem (COFEN). Resolução COFEN n 527 , de 10 de novembro de 2016. Atualiza e estabelece parâmetros para o dimensionamento do quadro de profissionais de enfermagem nos serviços/ locais em que são realizadas atividades de enfermagem [Internet]. 2016 [cited 2016 nov 28]. Available from: http://www.cofen.gov.br/resolucaocofen-no-05272016_46348.html

7. Conselho Federal de Enfermagem (COFEN). Resolução COFEN n 374, de
23 de março de 2011. Normatiza o funcionamento do sistema de fiscalização do exercício profissional da enfermagem e dá outras providências. [Internet]. 2011 [cited 2014 dec 12]. Available from: http://cofen.gov.br/ resoluo-cofen-3742011_6590.html

8. Conselho Federal de Enfermagem (COFEN). Lei no 7.498, de 25 de junho de 1986. Dispõe sobre a regulamentação do exercício da Enfermagem e dá outras providências [Internet]. 1986 [cited 2014 mar 05]. Available from: http://cofen.gov.br/lei-n-749886-de-25-de-junho-de-1986_4161.html

9. Conselho Federal de Enfermagem (COFEN) - Decreto Lei no 94.406 de 8 de junho de 1987. Regulamenta a Lei no 7.498, de 25 de junho de 1986, que dispõe sobre o exercicio da Enfermagem, e dá outras providências [Internet]. 1987 [cited 2014 mar 05]. Available from: http://www.cofen.gov. br/decreto-n-9440687_4173.html

10. Agência Nacional de Vigilância Sanitária. Resolução Da Diretoria Colegiada. RDC n 26 de 11 de maio de 2012. Altera a Resolução RDC 7 de 24 de fevereiro de 2010 que dispõe sobre os requisitos mínimos para funcionamento de unidades de terapia intensiva e da outras providências. [Internet]. 2012 [cited 2014 June 10]. Available from: http://bvsms.saude. gov.br/bvs/saudelegis/anvisa/2012/rdc0026_11_05_2012.html

11. Martinato MCNB, Severo DF, Marchand EAA, Siqueira HCH. Absenteismo na enfermagem: uma revisão integrativa. Rev Gaúcha Enferm. [Internet]. 2010 [cited 2014 June 10];31(1):160-6. Available from: http://www.scielo.br/ pdf/rgenf/v3lnl/a22v3lnl.pdf

12. Perez Junior EF, Oliveira EB, Souza NVDO, Lisboa MT, Silvino ZR. Segurança no desempenho e minimização de riscos em terapia intensiva: tecnologias duras. Rev. enferm. UERJ [Internet]. 2015 [cited 2015 Sep];22(3): 327-333. Available from: http://www.facenf.uerj.br/v22n3/v22n3aO6.pdf

13. Menegueti MG, Nicolussi AC, Scarparo, Campos LF, Chaves LDP, Laus AM. Dimensionamento de pessoal de enfermagem nos serviços hospitalares: revisão integrativa da literatura. Rev. Eletr. Enf. [Internet]. 2013 [cited 2014 June 10]:15(2):551-62. Available from: http://www.fen.ufg. $\mathrm{br} / \mathrm{fen} \_$revista/v15/n2/pdf/v15n2a30.pdf 